\title{
Genetics-guided treatment for chronic pain
}

Efklidis Raptis ${ }^{1, *}$

${ }^{1}$ Medical Effectiveness \& Obesity, South \& East Europe, Middle East, Novo Nordisk, Africa. efklidis.raptis@gmail.com

Chronic pain treatment is often compromised by adverse reactions, low efficacy, and potentially dangerous drug interactions. Genetics may, in some cases, help avoid such issues and it is conceivable that, in the near future, the treatment of chronic pain will be guided -at least in part- by the genetic background of individual patients. A typical example is the polymorphisms of the cytochrome enzymes (e.g., P450); understanding their impact on substrate metabolism can significantly help to avoid lack of efficacy and adverse events for medications often used in the treatment of chronic pain, such as opioids, NSAIDs and membrane stabilizers (antiepileptics). There is abundant literature on other relevant examples, such as receptors polymorphisms (e.g., OPRM1), HLA genotypes (e.g., HLA-A*31:01), enzyme and transporter polymorphisms (e.g., COMT, UGT, ABCB1), cytokine profiles ( $\pi \cdot \chi$. IL-6), ion channel and transcriptional factor polymorphisms etc. Current research, using data from channelopathies and ion channel mutations related to pain transduction and conduction, attempts to develop treatments for chronic pain syndromes which will utilize a guided and individualized approach to achieve safer and more efficacious therapies. Accordingly, genetics has allowed for the identification of novel modes of action for old compounds already used for the treatment of chronic pain. By combining pharmacogenetics, proteomics, epigenetics and neurophysiology, it is foreseeable that we will manage to approach the underlying pathophysiology of chronic pain in an individualized manner and, consequently, to adapt the treatment. In addition, genetic therapy (e.g., RNAi, ASOs, CRISPRi-KRAB) may further help manage difficult chronic pain syndromes. There are still many barriers to overcome, such as the availability of genetical analytics and their performance, the costeffectiveness ratio, the lack of relevant data from randomized controlled clinical trials, as well as the limited number of approved treatments with different modes of action for chronic pain. 